\section{Patient-Ventilator Asynchrony and Standard Waveforms: Looks Can Be Deceiving}

\section{To the Editor:}

In a recent issue of ResPIRATORY CARE, Ramirez et al $^{1}$ assessed clinicians' ability to detect patient-ventilator asynchrony using standard ventilator waveforms. I applaud their contribution. The ability to interpret ventilator waveforms is essential for safe and effective ventilator management. The authors effectively articulate the importance of identifying and minimizing patient-ventilator asynchrony. The purpose of this letter is not to criticize the contribution of Ramirez et al, ${ }^{1}$ but rather to take the opportunity to call attention to the fact that in terms of patient-ventilator asynchrony, standard ventilator waveforms can be quite deceiving. Indeed, marked patient-ventilator asynchrony can be accompanied by satisfactory waveforms. A more complete patient-ventilator synchrony assessment requires the addition of diaphragmatic electromyogram waveforms. ${ }^{2}$ Figure 1 shows the standard pressure, flow, and volume versus time waveforms in a patient with severe COPD. The standard waveforms do not indicate any significant asynchrony. However, when you superimpose the diaphragmatic electromyogram tracing over the pressure versus time waveform, marked inspiratory trigger asynchrony can be appreciated. In addition, the diaphragmatic electromyogram peak value of $38 \mu \mathrm{V}$ indicates that the patient is experiencing a fatiguing work load, which is due in some part to imposed work of breathing, a consequence of inspiratory trigger asynchrony. Standard ventilator waveforms without diaphragmatic electromyogram limit the ability of clinicians to detect patient-ventilator asynchrony.

Jeffrey M Haynes RRT RPFT FAARC Respiratory Therapy Department St Joseph Hospital Nashua, New Hampshire

Mr Haynes has disclosed a relationship with Morgan Scientific.

DOI: $10.4187 /$ respcare.05593

\section{REFERENCES}

1. Ramirez II, Arellano DH, Adasme RS, Landeros JM, Salinas FA, Vargas AG, et al. Ability of ICU health-care professionals to identify patient-ventilator asynchrony using

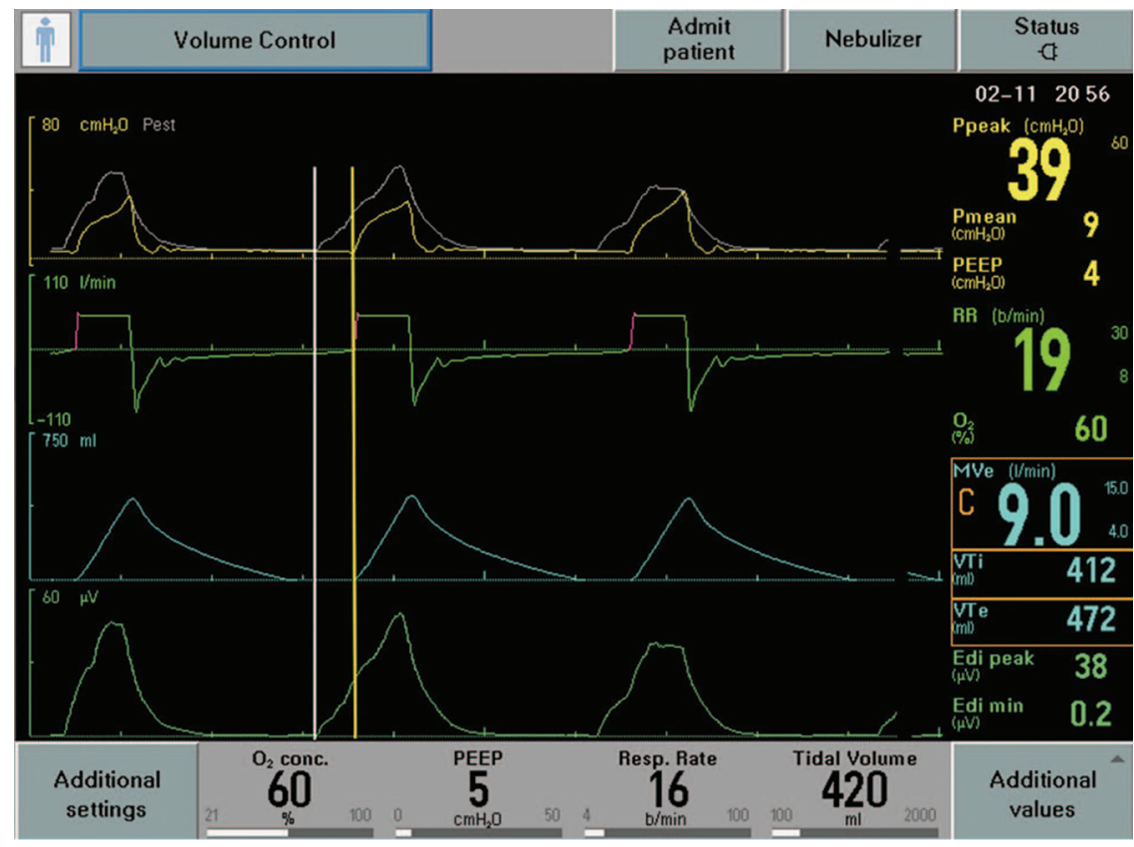

Fig. 1. Ventilator waveforms from a patient with severe COPD. The tracings represent pressure (yellow), flow (green), volume (blue), and diaphragmatic electromyogram (Edi, white) versus time. The white vertical line indicates the onset of diaphragmatic electromyogram activity, and the yellow vertical line indicates the onset of ventilator response via flow triggering.

waveform analysis. Respir Care 2017;62(2): 144-149.

2. Verbrugghe W, Jorens PG. Neurally adjusted ventilatory assist: a ventilation tool or a ventilation toy? Respir Care 2011;56(3):327-335.

\section{Patient-Ventilator Asynchrony and Standard Waveforms: Looks Can Be Deceiving-Reply}

In reply:

We sincerely thank Jeffrey Haynes for his comments on our study. ${ }^{1}$ We agree with Mr Haynes when he points out that there are occasions in which patient-ventilator asynchrony cannot be identified by standard waveform analysis and that other assessment tools, such as diaphragmatic electromyography, are needed to effectively identify its presence. This is a very important point, considering that a significant percentage of asynchronies are underestimated using standard waveform analysis. However, identifying patient-ventilator asynchrony using waveform analysis is an available tool in all modern mechanically ventilators that has been shown to have good correlation with other methods, such as diaphragmatic pressure measurement. ${ }^{2}$

It is also important to consider that diaphragmatic electromyography has proved to be a very useful tool to obtain very interesting results from the physiological point of view, ${ }^{3}$ but it is not frequently used during daily clinical practice to identify patientventilator asynchrony in mechanically ventilated patients in the ICU. It could be an interesting idea to develop some cost-effectiveness studies in which we could investigate whether reducing patient-ventilator asynchrony through correct interpretation of standard waveform analysis improves clinical outcomes with regard to incremental cost-effectiveness ratios. Finally, it is important to remember that the main objective of our study was not to determine the best method or tool to identify patient-ventilator asynchrony, but to assess the ability of ICU health-care professionals to identify patientventilator asynchrony using standard waveform analysis.

Ivan I Ramirez PT

Division of Critical Care Medicine Hospital Clínico Universidad de Chile Santiago, Chile Universidad de los Andes Santiago, Chile

Daniel H Arellano PT CRT MSc

Division of Critical Care Medicine Hospital Clínico Universidad de Chile Santiago, Chile 